\title{
Research on Government Purchase Public Service Model Based on SCOR Model
}

\author{
Yawei Cao, Jie Cai \\ Ocean University of China, Qingdao 266100, China
}

Keywords: Supply chain management; SCOR model; government purchase; public service

\begin{abstract}
Based on the analysis of the status quo of the government's purchase of public services in China, this paper combs the process of government's purchase of public services, and puts forward the SCOR model based on ternary structure in line with the present situation of government's purchase of public services. The purchase of public services provides a theoretical basis for the successful development of our country.
\end{abstract}

\section{基于 SCOR 模型的政府购买公共服务模型研究}

\author{
曹亚玮，蔡婕 \\ 中国海洋大学管理学院, 山东青岛, 266100
}

\begin{abstract}
摘要: 本文在对政府购买公共服务在我国的发展现状进行分析的基础上，对我国政府购买公共服务的流程 进行梳理, 提出符合我国政府购买公共服务现状的基于三元结构的 SCOR 模型, 为政府购买公共服务在我 国的成功发展提供理论基础。
\end{abstract}

关键词：供应链管理; SCOR 模型; 政府购买; 公共服务

一. 问题的提出

随着供应链理论的不断完善和发展, 企业对供应链的关注也由生产性供应链扩展到服务供应链, 然而 涉及到公共部门的服务供应链研究却略显不足。本文尝试对供应链运作参考模型 (SCOR) 进行改进并结合 服务供应链相关理论来对政府购买公共服务进行流程分析, 期望从中找到适用于中国政府购买公共服务的 模型基础。

\section{二. 相关理论概述}

自 Lisa. M. E11ram 在 2004 年发表 “理解和管理服务供应链” 一文, 首次提出服务供应链的概念, 服务 供应链逐渐引起国内外学术研究的关注。一部分国内学者开始以特定服务行业为背景, 对存在于这些领域 的服务供应链进行研究。李德生对电子商务服务平台中即时构建服务供应链关键问题进行研究 ${ }^{[1]}$; 邵万清 分析了港口服务供应链的协调机制 ${ }^{[2]}$; 朱荣艳以系统的视角对航运物流服务供应链的协同优化展开研究 [3]。

基于上述学者的研究, 本文梳理出服务供应链与生产性供应链的主要几个不同点, 如表 1 所示。

另有学者侧重于研究服务供应链管理模式, 并尝试定义和构建服务供应链基本框架。付秋芳、王文博 提出了服务供应链运作参考模型 (SSCOR) 并给出其 $1 、 2 、 3$ 层运作模式 ${ }^{[4]}$; 卢忠东运用 SCOR 模型和服务 质量差距模型分析客户服务流程, 提出服务供应链战略管理基本框架 ${ }^{[5]}$; 单泪源等在分析 E11ram 专业服 务供应链模型和 IUE-SSCM 模型基础上提出了服务供应链拓展模型 ${ }^{[6]}$; 简兆权等提出一个服务供应链整合与 服务创新概念模型 ${ }^{[7]}$ 。

本文通过综合已有的服务供应链运行模式, 归纳出一个简化的服务供应链模型, 如图 1 。

服务供应链主体: 功能型服务提供商 (Service Provider) -服务集成商 (Service Integrators)-市场 客户 (Market Customers)。 
表 1. 生产性供应链与服务供应链的比较

\begin{tabular}{|l|l|l|}
\hline \multicolumn{2}{|c|}{ 生产性供应链与服务供应链的比较 } \\
\hline 类别 & 生产性供应链 & 服务供应链 \\
\hline 交易主体 & 实物产品 & 服务 \\
\hline 运作模式 & 推动型和拉动型 & 市场反应、完全拉动 \\
\hline 稳定性 & 良好的系统稳定性 & 异质化的客户需求, 不确定性较大 \\
\hline 管理重点 & 预测顾客需求并通过 & 对服务能力、服务交付的管理, 创造 \\
& 生产计划、库存管理等 & 并满足客户的多样化需求 \\
\hline 绩效评价 & 来实现生产 & \\
\hline
\end{tabular}

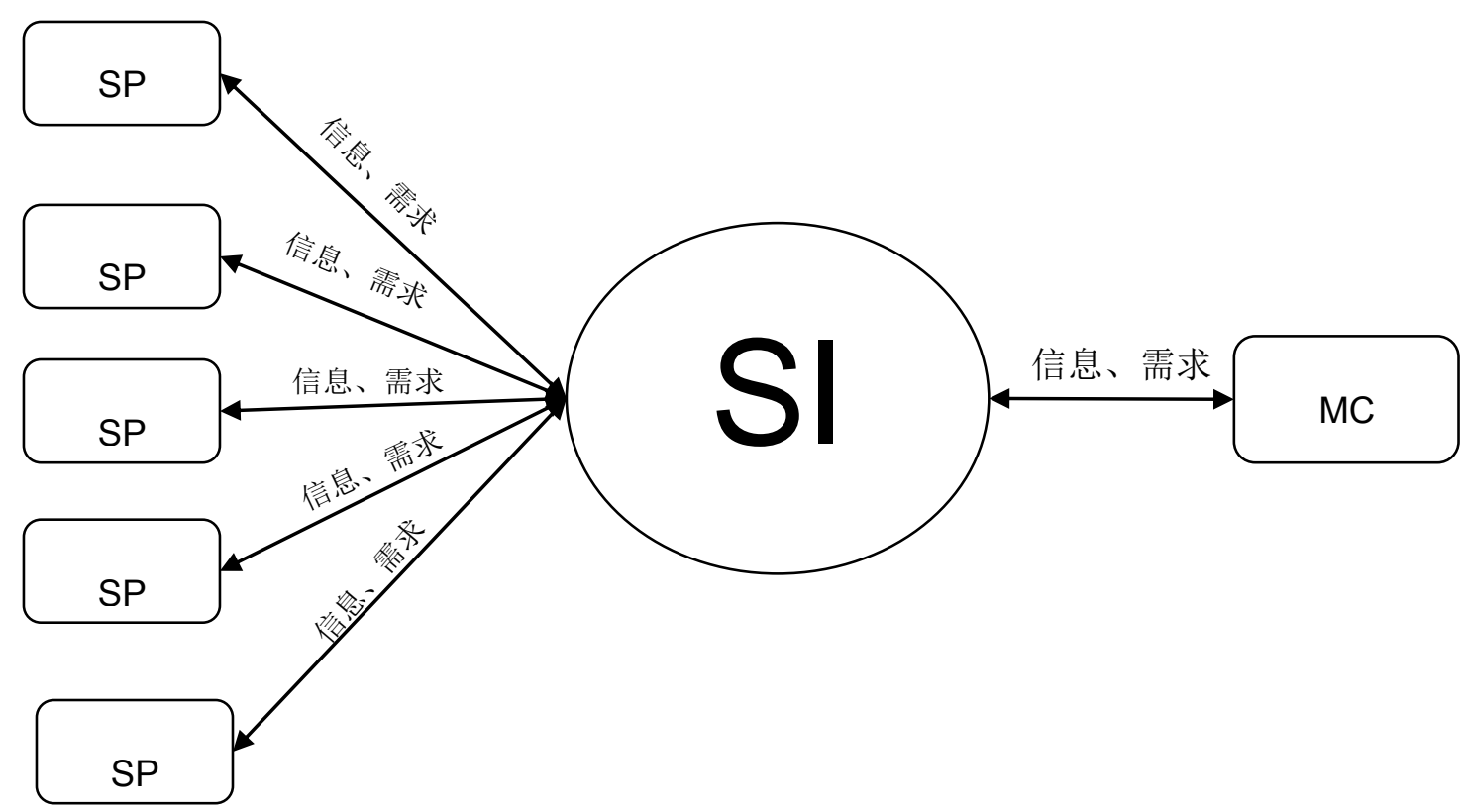

图 1. 简化的服务供应链模型

\section{三. 政府购买公共服务形成的供应链}

\section{（一）改进的 SCOR 模型}

由于 SCOR 模型是制造行业的通用模型, 缺乏行业针对性, 本文对 SCOR 模型进行改进, 流程之间的对 应关系如表 2 所示: 
表 2. 改进的 SCOR 模型的流程

\begin{tabular}{|l|l|}
\hline SCOR 模型的流程 & 改进的 SCOR 模型的流程 \\
\hline 计划 & S1 计划 \\
\hline 采购原材料 & S2 招标, 寻找服务供应者 \\
\hline 制造 & S3 服务供应者筹建项目 \\
\hline 配送 & S4 供给服务 \\
\hline 退货 & $\begin{array}{l}\text { S5 服务供应链不涉及退货, 改为 } \\
\text { 反馈 }\end{array}$ \\
\hline 支持 & S6 支持 \\
\hline
\end{tabular}

\section{（二）基于三元结构的政府购买公共服务模型}

\section{1. 利益相关者分析}

1. 1. 政府

政府是公共服务的主导者、协调者和整合者。政府作为公共服务购买的服务集成商，其扮演的角色贯 穿整个购买服务过程的始终，在 SCOR 模型中涉及 S1、S2、S3、S4、S5、S6 全部环节。

1. 2. 直接服务供应者

在我国最初政府购买公共服务的对象多为社会组织，但是自 2012 年以来，国务院出台了一系列鼓励 社会资本参与到政府购买公共服务项目中来的政策, 调动了企业参与公共服务的热情。其在 SCOR 模型中 处于 S2、S3、S4、S5 环节。

1. 3. 社会公众

社会公众是公共服务的需求方。在政府购买公共服务的整个流程中，一方面，公民向政府表达合理需 求; 另一方面, 社会公众不断参与事前、事中, 特别是事后的体验反馈。其参与 SCOR 模型中的 S1、S4、 S5 环节。

1.4. 间接服务供应者

间接服务供应者为直接服务提供者有偿提供物质和资源, 一般是市场体系中的盈利性企业, 比如地铁 项目中的建设方等。其主要在 SCOR 模型中的 S3 环节发挥作用, 但如果涉及提供的物质、工程质量问题的 反馈，S5 环节中必须将其包含进来。

\section{2. 基于三元结构的政府购买公共服务模型 （图 2）}

本文初步提出一个基于三元结构的政府购买公共服务模型。由图 2 可知, 公共服务模型中以公共服务 为主导, 伴随着信息和资金的流动, 强调整体的价值增值和供需均衡。

\section{3. 基于三元结构的政府购买公共服务模型}

\section{1 定义层}

模型的底层是定义层，初步确立了政府购买服务的基本范围和内容，第一层共有计划、购买、建设、 供给、反馈、支持六个基本流程（如图 3):

\section{1.1 计划}

政府根据社会公众的需求、服务供应商的服务能力、社会经济环境等条件, 制定采购目标和实现该目 标的途径。包括 P1 供应链计划、P2 购买计划、 P3 供给计划、P4 反馈计划、P5 支持计划。

\section{1.2 购买}

政府在收集社会公众需求、评估服务供应商服务能力的基础上，以公开招标、竞争性谈判等方式选择 资质和信誉好的服务供应商, 获取服务。包括 B1 公众需求分析、B2 采购方案设计、B3 供应商选择。

\section{1.3 建设}

承接公共服务项目的社会组织或企事业单位，按照与政府事先签订的合同要求，自己建设或寻找第三 方组织建设提供相应公共服务的必要基础设施和场所, 保障公共服务的顺利供给。包括 C1 公共服务项目 分析、C2 间接服务提供者选择、C3 项目完工评审验收。 


\section{1.4 供给}

社会组织、事业单位或企业作为直接服务提供者，在必要的基础设施完工并且通过审验确定可以投入 使用后，按照事先与政府签订的合同进入向社会公众提供公共服务、满足公众需求、进行服务质量控制与 监督的过程，包括：S1 服务交付、S2 服务质量控制等。

\section{1 .5 反馈}

社会公众对服务质量进行评价并反馈给政府和直接服务提供者，服务提供者进行质量评估并改进提高 服务水平和质量的过程。包括 F1 信息反馈、F2 补救管理、F3 客户关系管理。

3. 1.6 支持

支持流程与供应链管理密切相关、贯穿始终，支持服务供应链的计划制定和执行，对绩效、资源、合 同、风险等方面进行有效管理，来确保每个流程正常运行。包括 E1 计划支持流程、E2 购买支持流程、E3 建设支持流程、E4 供给支持流程、E5 反馈支持流程。

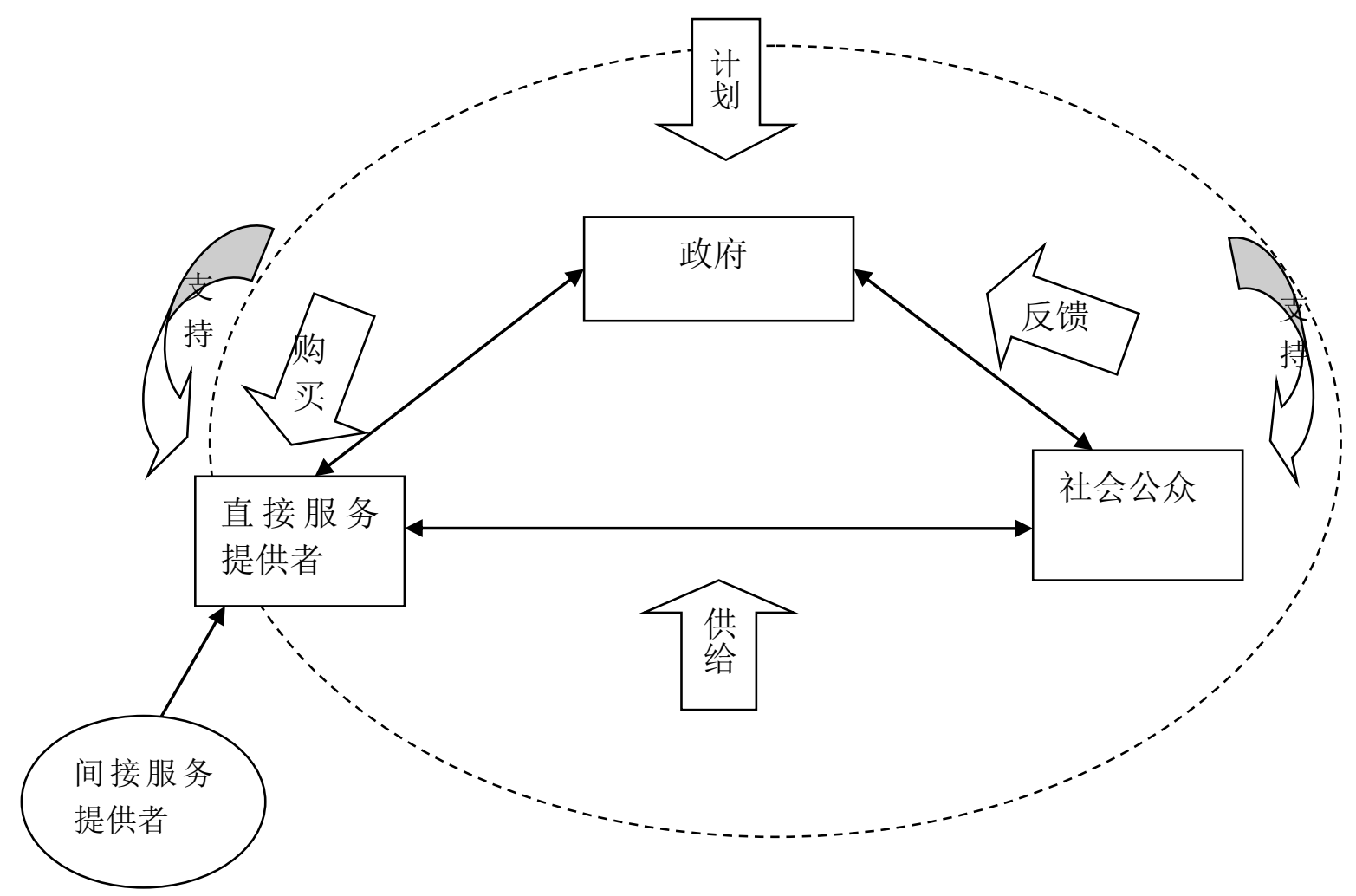

图 2. 基于三元结构的政府购买公共服务模型 


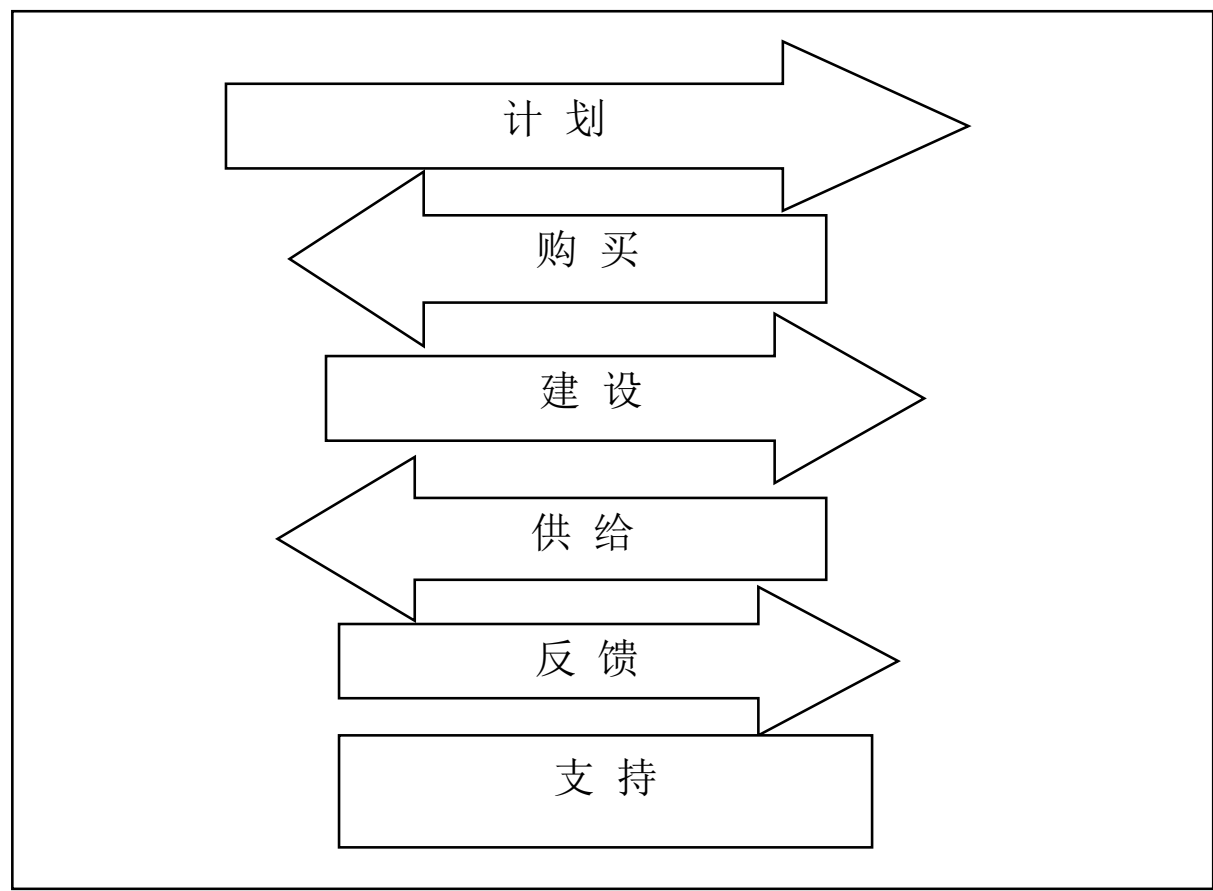

图 3. 基于三元结构的政府购买公共服务模型定义层运作模式

\section{2. 配置层}

配置层处于该模型的第二层, 是对第一层基本流程的分解与展开。配置层定义了三种流程类型, 分别 是计划类流程、执行类流程和支持类流程。

表 3. 核心流程的种类

\begin{tabular}{|l|l|l|l|l|l|l|l|}
\hline \multicolumn{2}{|c|}{} & & \multicolumn{5}{|c|}{ SC0R 模型 } \\
\cline { 3 - 9 } \multicolumn{2}{|c|}{} & P 计划 & B 购买 & C 建设 & S 供给 & F 反馈 & E 支持 \\
\hline \multirow{2}{*}{$\begin{array}{l}\text { 流程 } \\
\text { 型 }\end{array}$} & 计划类 & P1-P5 & & & & & \\
\cline { 2 - 8 } & 执行类 & & B3 & C1-C3 & S1 & F2、 F3 & \\
\cline { 2 - 8 } & 支持类 & & B1、 B2 & & S2 & F1 & E1-E4 \\
\hline
\end{tabular}

\section{(1) 计划类流程}

计划类流程是指在特定的时间和空间范围内确定一系列活动，即有计划地收集各类信息、协调各种资 源满足供应链客户的需求。

表 4. 计划类核心流程

\begin{tabular}{|l|l|}
\hline \multicolumn{2}{|c|}{ 计划类核心流程 } \\
\hline \multicolumn{1}{|c|}{ 流程名称 } & 定义 \\
\hline P1 供应链计划 & 政府根据社会公众需求、服务供应商特点进行总体规划的过程 \\
\hline P2 购买计划 & 政府确定资质和信誉好的服务供应商的计划过程 \\
\hline P3 供给计划 & 服务提供商向社会公众提供服务产品进行计划的过程 \\
\hline P4 反馈计划 & 政府和服务提供者对从社会公众搜集而来的评估、反馈信息的计划过程 \\
\hline P5 支持计划 & 确保服务供应链各个流程顺利运行所制定计划的过程 \\
\hline
\end{tabular}

(2) 执行类流程

执行类流程指按照公众需求和服务计划实施服务直到完成服务的整个过程。 
表 5. 执行类核心流程

\begin{tabular}{|c|c|}
\hline \multicolumn{2}{|r|}{ 执行类核心流程 } \\
\hline 流程名称 & 定义 \\
\hline B3 供应商选择 & 政府机构选择资质信誉好的服务提供商 \\
\hline $\begin{array}{l}\text { C1 公共服务项目 } \\
\text { 分析 }\end{array}$ & 直接服务提供者对此次承接的公共服务项目进行分析, 确定服务提供方案 \\
\hline $\begin{array}{l}\mathrm{C} 2 \text { 间接服务提供 } \\
\text { 者选择 }\end{array}$ & $\begin{array}{l}\text { 直接服务提供者选择资质信誉好的间接服务提供者承建提供公共服务所需要的基础设 } \\
\text { 施, 并制定相关合同。在此环节, 间接服务提供者开始参与到整个流程中来 }\end{array}$ \\
\hline $\begin{array}{l}\text { C3 项目完工评审 } \\
\text { 验收 }\end{array}$ & $\begin{array}{l}\text { 间接服务提供者将承建的项目交付给直接服务提供者, 直接服务提供者对其进行评审 } \\
\text { 验收, 确定其符合要求 }\end{array}$ \\
\hline S1 服务交付 & $\begin{array}{l}\text { 服务提供商根据其拥有的服务能力, 按照与政府签订的合同要求向社会公众提供服务 } \\
\text { 产品 }\end{array}$ \\
\hline F2 补救管理 & 服务供应商对提供服务过程中出现的服务失败失误情况采取措施进行挽救 \\
\hline F3 客户关系管理 & 服务供应商在服务过程中改善与社会公众的关系, 提升服务提供水平 \\
\hline
\end{tabular}

(3) 支持类流程

支持类流程主要对其他流程起到维护、支持、管理的作用，服务于政府购买的整个过程。 表 6. 支持类核心流程

\begin{tabular}{|l|l|}
\hline \multicolumn{2}{|c|}{ 支持类核心流程 } \\
\hline \multicolumn{1}{|c|}{ 流程名称 } & 定义 \\
\hline B1 公众需求分析 & 政府收集、分析社会公众对公共服务产品的需求 \\
\hline B2 采购方案设计 & 政府根据公众需求分析的结果设计合理的采购方案 \\
\hline S2 服务质量控制 & 服务提供者监督服务提供过程, 消除公众不满意现象, 提高公众满意度 \\
\hline F1 信息反馈 & 社会公众对购买服务产品中的满意度进行反馈, 服务提供者进行反馈收集 \\
\hline E1一E4 支持流程 & 对基本流程的支持和维护过程 \\
\hline
\end{tabular}




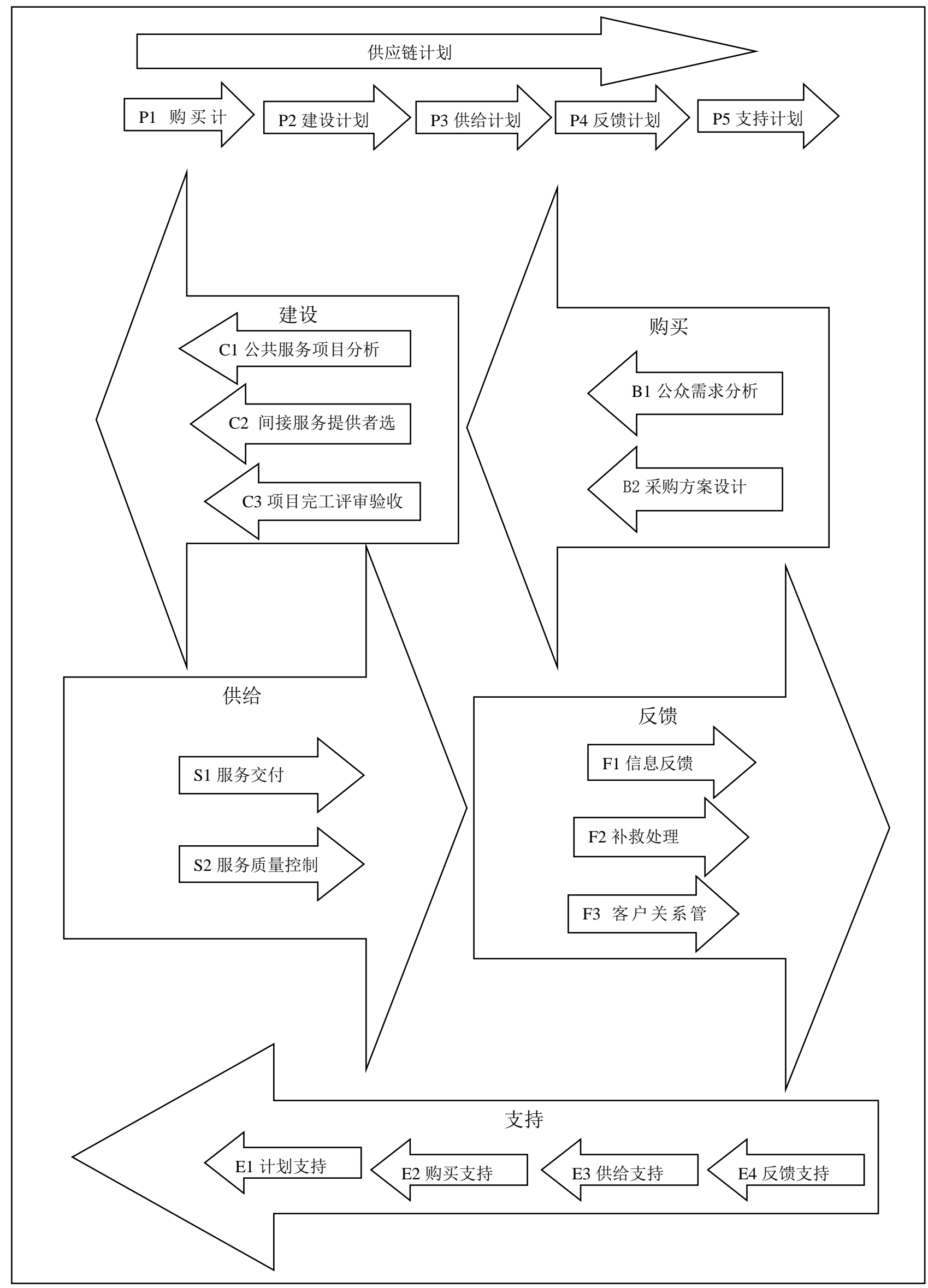

图 4. 基于三元结构的政府购买公共服务模型配置层运作模式 


\section{3. 流程因素层}

模型第三层流程因素层是对第二层配置层的进一步细分，为供应链的运作提供更详细的信息。同时， 流程因素层还涉及相关的输入和输出信息, 如 F2.2 制定服务改进方案, 其中输入的信息涉及公众提供的 反馈信息等。

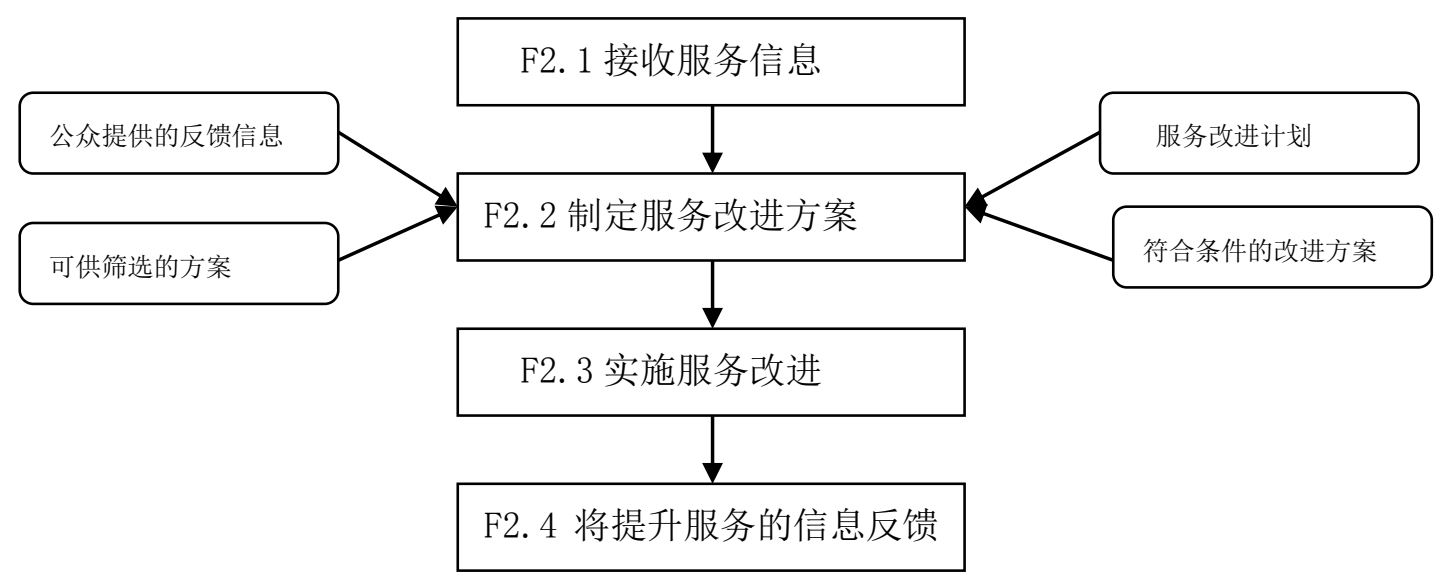

图 5. 流程元素层运作模式 (以 F2 补救管理为例)

关于模型第四层及以下实施层的构建, 本文不再进行具体讨论。

\section{四. 结论}

尽管本文提出了基于三元结构的政府购买公共服务模型，但是仍存在着一定的局限性。首先，该模型 仅仅是在理论层面上对于政府购买公共服务的流程进行分析和定义，尚未应用到实际的政府购买公共服务 业务中，缺乏实证的支持。其次，该模型仅初步定义了一、二、三层的运作模式，在绩效评估和风险识别 方面还有待完善。今后的研究中需要结合实际购买

服务的类型对该模型进行验证和改进，逐步完善政府购买服务理论体系，同时进一步完善模型绩效评 估和风险识别模块，对评价指标体系的设计、指标的度量和量化、绩效评价方法等进行进一步讨论，建立 一套完整的、能够为政府购买不同类型的公共服务建立模型时提供范本的模型基础，帮助政府对其购买流 程进行规范，为各地方政府购买公共服务时提供理论依据。

\section{References}

[1] Li Desheng. E-commerce service platform in the immediate construction of the key issues of service supply chain [D]. Shandong University 2012.

[2] Shao Wanqing. Port Service Supply Chain Coordination Mechanism [D]. Donghua University 2013.

[3] Zhu Rongyan. Shipping logistics service supply chain collaborative optimization [D]. Wuhan University of Technology 2014.

[4] FU Qiu-fang, WANG Wen-bo.Study on Service Supply Chain Operation Reference Model [J]. Logistics Technology. 2010 (16).

[5] Lu Zhongdong. Service supply chain nature analysis and operational framework conceptual model construction $[\mathrm{J}]$. Business era. 2012 (24).

[J]. Science and Technology Progress and Countermeasures. 2011 (21) [J]. Science and Technology Progress and Countermeasures. [J]. Science and Technology Progress and Countermeasures. $2011(21)$.

[7] Jane Zhaoquan, Li Lei, Liu Yi. Service supply chain integration and its impact on service innovation review and outlook [J]. Foreign Economy and Management. 2013 (01). 